\title{
Central cannulation is safe in acute aortic dissection repair
}

\author{
T. Brett Reece, MD, Curtis G. Tribble, MD, Robert L. Smith, MD, R. Ramesh Singh, MD, Brendon M. Stiles, \\ Benjamin B. Peeler, MD, John A. Kern, MD, and Irving L. Kron, MD
}

Objective: The site of cannulation for the repair of ascending aortic dissection remains controversial. It is not clear whether cannulation of the dissected vessel is safe or even preferred. We hypothesized that cannulation of the dissected aorta could be done safely with acceptable complication and mortality rates in this high-risk population.

Methods: The charts of repairs of acute ascending aortic dissections $(n=70)$ from 1996 to 2005 were reviewed. Cannulation was accomplished in 24 patients via the dissected aorta (central) and in 46 patients through cannulation of the femoral or axillary artery (peripheral). All were converted to sidearm cannulation of the graft for reperfusion. Groups were compared on the basis of comorbidities in addition to mortality, complications, hospital stays and final disposition.

Results: The groups were comparable on the basis of age and preoperative comorbidities. Similarly, there were no differences in bypass time, crossclamp time, or hypothermic circulatory arrest time between groups. Hospital mortality and postoperative complications, including stroke, were similar between groups, but the peripheral group experienced more cardiac events (peripheral 15\% vs central 0\%; $P<.05$ ) and higher mortality than the central group (peripheral $19.5 \%$ vs central $4.2 \% ; P<.05)$.

Conclusions: Direct cannulation of the dissected aorta was safe compared with peripheral cannulation in these patients. Inasmuch as these data demonstrate that cannulation of the dissected ascending aorta is safe, this technique can be used to tailor the cannulation approach to specific anatomic and patient characteristics that might optimize postoperative outcomes in this disease entity.

From the University of Virginia, Department of Surgery, Division of Thoracic and Cardiovascular Surgery, Charlottesville, Va.

Read at the Thirty-second Annual Meeting of the Western Thoracic Surgical Association, Sun Valley, Idaho, June 21-24, 2006.

Received for publication June 17, 2006; revisions received Sept 9, 2006; accepted for publication Sept 20, 2006.

Address for reprints: T. Brett Reece, MD, University of Virginia Health System, Department of Surgery, PO. Box 801359, MR4 Building, Room 3116, Charlottesville, VA 22908 (E-mail: tbr5q@virginia.edu).

J Thorac Cardiovasc Surg 2007;133:428-34 $0022-5223 / \$ 32.00$

Copyright () 2007 by The American Association for Thoracic Surgery

doi:10.1016/j.jtcvs.2006.09.059
$\mathrm{T}$ he optimal management of acute dissection of the ascending aorta remains surgical. ${ }^{1,2}$ Historically, morbidity from this entity has been reported as $30 \%$ or higher, but more recently several centers have published mortalities that can approach $10 \% .^{3-6}$ Although outcomes of these surgically treated patients have improved, the optimal approach to cannulation for these cases is not known.

Currently, three cannulation options exist. First, cannulation using the common femoral artery is one standard option. Despite widespread use of this route, femoral cannulation can carry some risk of critical organ malperfusion, retrograde embolization, and femoral arterial injury. Second, axillary cannulation has more recently become a widely used approach for arterial cannulation, especially in ascending aortic and arch surgery. There are also drawbacks to the use of this approach, including the extra time that is required for sewing a graft or repairing the axillary artery. Third, direct cannulation of the dissected ascending aorta has been used occasionally, but has been mainly reported as a bail-out technique when other cannulation options are not available. ${ }^{7-9}$ Although this technique allows for cannulation of a part of the vessel that will be excised during the repair, some argue, 
without data, that this technique may risk rupture, extension of the dissection, or embolization of debris into cerebral or solid organ vasculature beds.

For the purpose of this study, we hypothesized that direct cannulation of the dissected ascending aorta was at least as safe as peripheral cannulation through the femoral or axillary arteries for cases of acute ascending aortic dissection. Furthermore, on the basis of our data, we hope to propose a system for choosing the optimal cannulation site given the specific dissection characteristics and patient attributes.

\section{Materials and Methods}

After gaining approval for this study from the Human Investigation Committee at the University of Virginia (with waiver of consent), all acute ascending aortic dissections were identified from our retrospectively collected aortic database including cases performed between July 1996 and July 2005. From more than 800 aortic cases, 70 acute ascending aortic dissections that were operated on were identified. These patients were then further divided into two groups and compared on the basis of the initial site of cannulation, including central (cannulation of the ascending aorta) and peripheral (cannulation of the femoral or right axillary artery). These groups were compared on the basis of preoperative, intraoperative, and postoperative characteristics.

Although some variability occurred during the study period, most patients were cared for in a similar fashion. Various diagnostic radiologic techniques were used including angiography, echocardiography, computed tomographic angiography, and magnetic resonance angiography. More recently, all patients had a computed tomographic angiogram for diagnosis and operative planning. Criterion for site of cannulation varied over time and among surgeons, but an operative plan for the site of cannulation was established before going to the operating room. The final determination of the suitability of the chosen cannulation site was made in the operating room after direct inspection of the vessels.

Although the sites of cannulation varied, the approaches to cooling and circulatory arrest were similar. The goal of ascending aortic dissection repair was prevention of proximal rupture and preservation of aortic valve competence. Thus, all procedures were planned for replacement of the ascending aorta with repair/replacement of the aortic valve as needed. The general approach to these patients included arterial cannulation of the chosen vessel and initiation of cardiopulmonary bypass. Most patients were slowly cooled to a core body temperature of $18^{\circ} \mathrm{C}$ to allow 20 to 30 minutes of circulatory arrest time. Only recently has antegrade perfusion started being used (in some axillary cannulations), but we generally have employed retrograde cerebral perfusion as previously described. ${ }^{10}$ Owing to fluctuations in practice over time, a variety of neuroprotective pharmacologic strategies have been used during this period, whereas electroencephalography, transcranial Doppler, and cerebral oximetry have been used inconsistently.

Patients undergoing femoral artery cannulation $(n=31)$ underwent femoral cutdown. A purse-string suture was placed on the anterior surface of the femoral artery. Through the purse-string suture, a cannula was placed by the Seldinger technique (Figure 1). In cases in which the vessel was calcified or appeared dissected, the artery was secured with vessel loops and vascular clamps, and
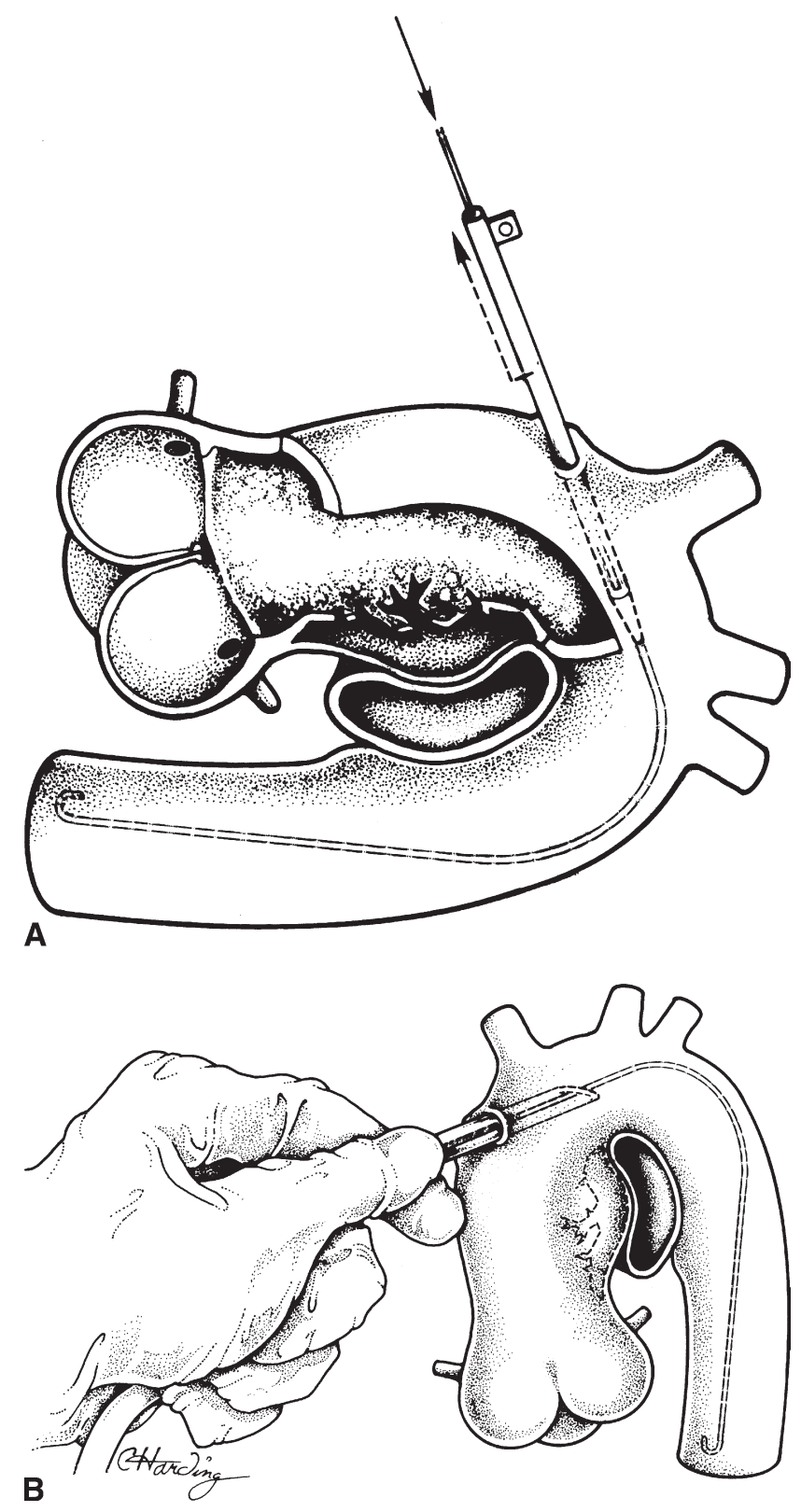

Figure 1. Cannulation of the ascending aorta. A, Gaining access to the lumen via the Seldinger technique with confirmation of wire placement in the descending aorta by transesophageal echocardiography. B, Placement of the cannula high in the ascending aorta with manual stabilization during cooling.

a transverse arteriotomy was made. The arteriotomies were closed primarily at the end of the procedure. Patients undergoing axillary cannulation $(\mathrm{n}=15)$ underwent axillary cutdown. The venous branches over the axillary artery were divided and the axillary vein was retracted out of the way. The axillary artery was looped and pulled up for the application of a Satinsky clamp. The arteriotomy was started with a knife and completed with an aortic punch used to make a circular hole to which an 8-mm Dacron graft was sewn. 
TABLE 1. Preoperative comorbidities

\begin{tabular}{|c|c|c|c|c|}
\hline Comorbidity & Central cannulation & Peripheral cannulation & $P$ value & Statistics \\
\hline Age & $61.8 \pm 3.2$ & $65.2 \pm 2.1$ & .39 & $t$ test \\
\hline Sex (female) & $8 / 24$ & $12 / 45$ & .56 & $\chi^{2}$ test \\
\hline DM & $3 / 24$ & $1 / 46$ & .11 & Fisher exact test \\
\hline Long-term steroid therapy & $4 / 24$ & $1 / 46$ & .04 & Fisher exact test \\
\hline CHF class & $2.9 \pm 0.2$ & $2.9 \pm 0.2$ & .84 & $t$ test \\
\hline CVA TIA & $4 / 24$ & $10 / 46$ & .77 & $\chi^{2}$ test \\
\hline PVD & $8 / 24$ & $16 / 46$ & .90 & $\chi^{2}$ test \\
\hline History of CAD & $7 / 24$ & $22 / 46$ & .13 & $\chi^{2}$ test \\
\hline Arrhythmia & $2 / 24$ & $7 / 46$ & .41 & $\chi^{2}$ test \\
\hline Marfan's & $1 / 24$ & $4 / 45$ & .65 & Fisher exact test \\
\hline Rheumatic & $1 / 24$ & $0 / 46$ & .34 & Fisher exact test \\
\hline CRI & $2 / 24$ & $2 / 44$ & .60 & Fisher exact test \\
\hline HD & $1 / 24$ & $0 / 46$ & .34 & Fisher exact test \\
\hline Emergency & $15 / 24$ & $34 / 46$ & .32 & $\chi^{2}$ test \\
\hline
\end{tabular}

The groups were similar in terms of all preoperative variables evaluated. $D M$, Diabetes mellitus; $C H F$, congestive heart failure; $C V A$, cerebrovascular accident; $T I A$, transient ischemic attack; $P V D$, peripheral vascular disease; $C A D$, coronary artery disease; $C R I$, chronic respiratory insufficiency; $H D$, heart disease; emergency, hypotensive, significant valvular insufficiency, pericardial effusion, coronary insufficiency, and aortic rupture.

Once peripheral cannulation had been achieved, cardiopulmonary bypass was initiated.

Cases involving central cannulation $(n=24)$ began with direct inspection of the ascending aorta after sternotomy and creation of the pericardial well. The only specific contraindication to this procedure was extensive hematoma in the wall of the aorta, ranging from thrombosed false lumen to intramural hematoma. The site of cannulation was determined from preoperative imaging as well as from intraoperative transesophageal echocardiography. Epicardial ultrasound was used if any question of clot in the vessel wall remained. Although cannulating the false lumen was not considered to be contraindicated, cannulation of the true lumen was preferred. The technique involves placement of a wire into the ascending aorta. Placement can be confirmed by identifying the wire in the descending thoracic aorta by transesophageal echocardiography. The site of cannulation was sufficiently high on the ascending aorta that a crossclamp could be placed more proximally in cases in which the vent was insufficient to overcome torrential aortic valve insufficiency, but low enough that the site of cannulation would be excised. Next, a percutaneous cannula was placed directly in the ascending aorta over the wire by the Seldinger technique with the cannulas loaded on a dilator (Figure 1). In almost all cases, the cannula was held in place by hand during cooling because of concerns about the ability of the dissected aorta to hold a purse-string suture. Once cardiopulmonary bypass was initiated, flow in both lumina were confirmed by transesophageal echocardiography. In all cases, the offending area of the ascending aorta was excised, and the distal anastomosis was created with a felt strip and a gel- or albumin-impregnated graft with a sidearm. After completion of this anastomosis, the sidearm of the graft was cannulated, cardiopulmonary bypass was restarted, and rewarming was begun. The aortic valve was resuspended when indicated and the proximal anastomosis was completed.

For this study, the type of cannulation, operative times (including hypothermic circulatory arrest time, crossclamp time, and cardiopulmonary bypass time), and any additional procedures were recorded. Surgeon preferences dictated the adjunct procedures, including coronary bypass, which was performed most commonly in cases of coronary dissection but also in cases of known significant coronary stenosis. Perioperative complications, hospital and intensive care unit lengths of stay, and follow-up were recorded and compared between groups. All statistics were performed by an independent statistician. Various techniques were used for comparing the groups, such as $\chi^{2}$ analysis, the Fisher exact test, and the Student $t$ test. The specific test used is noted for each comparision or group of comparisions.

\section{Results}

The two groups were similar with regard to preoperative comorbidities, as shown in Table 1. On the basis of these comparisons, the groups are valid for comparison. Several differences were identified in terms of procedures performed, as shown in Table 2. These differences may arise from increasing use of central cannulation at the same time as our increased comfort with valve preservation proce-

TABLE 2. Adjunct procedures

\begin{tabular}{lccl}
\hline Procedure & Central & Peripheral & $P$ value \\
\hline CABG & $6 / 24$ & $9 / 46$ & .6 \\
No. grafts/CABG & $10 / 6$ & $17 / 9$ & .8 \\
AVR & $4 / 24$ & $10 / 46$ & .6 \\
Valve resuspension & $11 / 24$ & $9 / 46$ & .03 \\
Root replacement & $11 / 24$ & $15 / 46$ & .3 \\
Any arch & $2 / 25$ & $12 / 46$ & $.045^{*}$
\end{tabular}

$C A B G$, Coronary artery bypass graft; $A V R$, aortic valve replacement. *The groups had similar procedures performed except that the peripheral group was significantly more likely to undergo replacement of some part of the aortic arch and less likely to undergo aortic valve resuspension. 
TABLE 3. Operative times

\begin{tabular}{lccc}
\hline & Central & Peripheral & $\boldsymbol{P}$ value \\
\hline HCA time & $35.1 \pm 3.8$ & $28.9 \pm 12.8$ & .162 \\
Crossclamp time & $114.4 \pm 13.0$ & $89.2 \pm 8.1$ & .158 \\
CPB time & $155.5 \pm 11.2$ & $180.3 \pm 8.6$ & .089 \\
\hline
\end{tabular}

In terms of operative times, there was no difference significant difference between groups regarding time on hypothermic circulatory arrest (HCA), crossclamp time, or cardiopulmonary bypass (CPB) time.

dures. Intraoperative times were similar between the groups, as demonstrated in Table 3.

In terms of complications and disposition, the groups were similar except that the peripheral cannulation groups had a siginificantly higher incidence of postoperative myocardial infarction as defined by significant troponin increase. These data are shown in Table 4. Similar rates of neurologic complications and hospital mortality were found, but significantly higher 30-day mortality was shown in the peripheral cannulation group compared with the central cannulation group. Of note, when comparing the small numbers of axillary and femoral cannulations, axillary cannulations had a higher 30-day mortality. These data show similar need for placement in a rehabilitation facility or skilled nursing facility between the two groups.

\section{Discussion}

The optimal cannulation site for the repair of ascending aortic dissection is not known. The most popular sites for cannulation in this setting are peripheral arteries such as the axillary artery or the femoral artery. ${ }^{11,12}$ A broad spectrum of approaches to these vessels has been published with satisfactory results. These variations include direct cannulation of the right axillary artery, sewing a graft to this vessel, and even perfusing via the carotid arteries. ${ }^{13-18} \mathrm{How}-$ ever, the cannulation of the dissected ascending aorta, or central cannulation, has not been used widely owing to concerns over the fragility of the vessel and over distal embolization. Interestingly, most cardiac surgical textbooks do refer to central cannulation as the "fallback" cannulation site in the setting of failure of primary cannulation attempts elsewhere. ${ }^{7-9}$

The purpose of this study was twofold: first, to demonstrate that cannulation of the dissected ascending is not only feasible, but safe; second, to show that this technique adds to the possible routes of cannulation for patients with ascending aortic dissections. The study was not intended to claim central cannulation to be the optimal approach in all patients presenting with dissection of the ascending aorta. Instead, by demonstrating that central cannulation was safe, we show that this approach could be added to the options available, providing data to help develop an alogorithm for which patient and dissection characteristics would be best served by each of the cannulation options.
TABLE 4. Complications and disposition

\begin{tabular}{lrlc}
\hline Complication/disposition & $\begin{array}{c}\text { Central } \\
\text { cannulation }\end{array}$ & $\begin{array}{c}\text { Peripheral } \\
\text { cannulation }\end{array}$ & $\begin{array}{c}\boldsymbol{P} \\
\text { value }\end{array}$ \\
\hline Infection & $21 \%(5 / 24)$ & $20 \%(9 / 46)$ & .8 \\
Pulmonary & $21 \%(5 / 24)$ & $28 \%(13 / 46)$ & .9 \\
Renal & $12.5 \%(3 / 24)$ & $17 \%(8 / 46)$ & .6 \\
Stroke/TIA & $21 \%(5 / 24)$ & $28 \%(13 / 46)$ & \\
Arrhythmia & $8 \%(1 / 24)$ & $15 \%(7 / 46)$ & .4 \\
MI & $0 \%(0 / 24)$ & $15 \%(7 / 46)$ & $<.01^{*}$ \\
Any cardiac & $12.5 \%(3 / 24)$ & $30 \%(14 / 46)$ & .07 \\
Other (ileus) & $4 \%(1 / 24)$ & $9 \%(4 / 46)$ & .4 \\
Any & $33 \%(8 / 24)$ & $51 \%(24 / 46)$ & .11 \\
Hospital mortality & $4 \%(1 / 24)$ & $20 \%(9 / 46)$ & .15 \\
$30-D a y$ mortality & $0 \%(0 / 24)$ & $17 \%(8 / 46)$ & $.04^{*}$ \\
Rehab/SNF placement & $26 \%(6 / 23)$ & $30 \%(11 / 37)$ & .8 \\
\hline
\end{tabular}

TIA, Transient ischemic attack; MI, myocardial infarction; Rehab, rehabilitation; SNF, skilled nursing facility. *Complications were similar between groups except that the peripheral cannulation group experienced significantly more perioperative myocardial infarctions and had a significantly higher 30-day mortality than the central cannulation group.

Although case numbers remain relatively small, several reports of cannulating the dissected ascending aorta, including this one, have now suggested that this approach is indeed safe. We and others have used central cannulation for nearly two decades, but the technique does not appear in the ascending aortic dissection literature until 1998 from an Italian group. ${ }^{19}$ This early experience suggested the feasibility of this technique. Minatoya and colleagues ${ }^{20}$ furthered this reported experience using central cannulation in 14 of 41 ascending aortic dissections over a 2-year period. They also believed that this approach provided more natural flow and avoided extension of the dissection. Our data support the findings of these previously reported and unreported series. In fact, these data imply that central cannulation may even be superior to peripheral cannulation in terms of lower postoperative mortality and fewer perioperative myocardial infarctions. We have several theories as to why this may be true. First, as postulated in the repair of ascending aortic aneurysms, the antegrade, or "natural," flow pattern is more likely to be preserved with central cannulation. Westaby and colleagues $^{21}$ studied central cannulation in arch and descending aortic aneurysms using circulatory arrest. They concluded that thoracoabdominal aortic perfusion through a femoral cannula predisposed patients to higher retrograde embolic risk. They suggested that cannulation of the ascending aorta close to the brachiocephalic vessel decreased this risk because of preservation of the natural blood flow pattern rather than a potentially more turbulent retrograde flow that may lift and embolize plaque. These principles could certainly translate to ascending aortic dissection. Moreover, central cannulation may decrease the incidence of malperfusion syndromes during cardiopulmonary bypass that can 
TABLE 5. Factors for choosing cannulation site

\begin{tabular}{lll}
\hline Cannulation site & \multicolumn{1}{c}{ Favorable characteristics } & \multicolumn{1}{c}{ Unfavorable characteristics } \\
\hline Central & $\begin{array}{l}\text { Type A dissection } \\
\text { Hemodynamic instability }\end{array}$ & $\begin{array}{l}\text { Thrombosed false lumen } \\
\text { Intramural hematoma }\end{array}$ \\
& Anticipated arch replacement & Antiph replacement \\
Axillary & Aissected arch aneurysm & Small or atherosclerotic vessel \\
& Type A dissection (confined to ascending aorta) & Hemodynamic instability \\
Femoral & Hemodynamic instability & Femoral dissection \\
& & Extensive peripheral vascular disease \\
& & Aortic atheroma \\
& & Obesity \\
\hline
\end{tabular}

be intrinsic to peripheral cannulation techniques. The institution of antegrade flow into the true lumen should, in theory, reduce the possibilities of distal malperfusion inasmuch as restoration of flow to the true lumen is the ultimate goal when treating complicated dissections of the descending aorta. Even when the false lumen is cannulated, the flow patterns are similar to those in the dissected state. The pressure is lower when pulsatile perfusion is abolished, minimizing the ongoing progression of the dissection.

Despite these favorable findings, these data are not meant to advocate central cannulation approaches over peripheral cannulation techniques. Instead, these data are intended to demonstrate that central cannulation is a safe option in some patients. The site of cannulation can be tailored to both the specifics of the dissection and the patient. For instance, there are cases in which central cannulation should be avoided. The risk of embolus with cannulation through thrombosed false lumen or intramural hematoma may make central cannulation prohibitive. Therefore, the presence and location of clot needs to be carefully considered, usually with a combination of preoperative and intraoperative radiologic imaging, echocardiography, and direct visualization. If clot is present, another cannulation approach should be considered. Futhermore, in cases that may involve more extensive arch work or longer hypothermic circulatory arrest time, the axillary approach may be more favorable than the central approach to allow antegrade cerebral perfusion techniques. Femoral cannulation may be relatively easy, with an easy vascular repair at the end of the case. But a subset of patients with aortic dissections extending distally or with extensive peripheral vascular disease may be more likely to experience malperfusion or arterial injury at the cannulation site. ${ }^{19,22,23}$ We try to avoid femoral cannulation in elderly patients with extensive aortic atheroma on preoperative imaging to avoid potential retrograde emoblization. Axillary cannulation has become increasingly popular recently in both ascending aortic dissections and ascending aortic aneurysms, especially those that may require some form of circulatory arrest. Strauch and colleagues ${ }^{11}$ from
Mount Sinai have suggested that axillary cannulation might be the optimal technique for reducing perfusion-related morbidity and adverse outcomes in both dissections and atherosclerotic aneurysms. Still, even in their deft hands, $5 \%$ of patients required alternative cannulation for various reasons or had complications attributable to the axillary cannulation, supporting our thought that no single approach is ideal for all patients. Specific patients in whom axillary cannulation may need to be avoided are those whose dissections extend into the axillary artery, those with an atherosclerotic axillary artery that may be prone to iatrogenic injury, those with a small axillary artery that may not support sufficient perfusion flow, those with vascular anomalies, and, finally, those with hemodynamic instability that may require more urgent initiation of cardiopulmonary bypass. ${ }^{24}$ On the basis of the specific aspects of the dissection anatomy and the patients' comorbidities, favorable sites for cannulation can be determined and unfavorable sites can be avoided to optimize potential outcomes, which are depicted in Table 5 .

We acknowledge that this study has its limitations. It is a single institutional retrospective study that can carry with it significant bias. Within the institution, though, various approaches were used, providing a mixture of techniques and philosophies for comparison. Despite these limitations, the cohorts did appear to be similar before the procedures. The total number of patients was small, which could create the potential for a type II error, but this is the largest number of cases published to date in which this technique was used. The limited number also forced comparison between central cannulation and two different peripheral cannulation techniques. The peripheral cannulation techniques described here are different from one another. However, they are currently the most commonly accepted sites of cannulation to which we can compare central cannulation for overall safety. We also concede that the strategies used for cerebral protection and monitoring, as well as radiographic evaluation over the time of this study, were not consistent but were similar between groups. 
Finally, the adjunct procedures were similar between the groups overall, but the central cannulation group underwent more valve resuspensions and fewer procedures involving the arch than the peripheral cannulation group. These differences could potentially confound our interpretation of the results. Since our intention was to demonstrate the safety of the technique rather than arguing that one approach is superior, we believe our conclusion remains valid despite these differences. Additionally, compared with other studies, our patients were more likely to undergo coronary revascularization. However, both the number of patients revascularized and the number of vessels revascularized per patient were similar between our groups. Despite these limitations, we believe these data demonstrate that central aortic cannulation of the dissected ascending aorta can be done safely when approached cautiously and meticulously.

In summary, these data have shown that central cannulation of the dissected ascending aorta can be performed safely. In particular, we found not only similar rates of neurologic complications, but also no difference in the need for placement in skilled nursing and rehabilitation facilities between the groups. Of note, the central cannulation group was less likely to have a postoperative myocardial infarction and had a lower 30-day mortality; however, hospital mortality was similar between groups. These results suggest that devotion to a single approach for cannulation in these cases can be avoided. Although all three options can be safely used, we believe that the site of cannulation should be tailored to each specific patient on the basis of patient characteristics and dissection anatomy. All three methods should be considered to optimize the care of these difficult patients. Although this study does not advocate using this approach on all cases of ascending aortic dissection, it does suggest that central cannulation can be used as safely as peripheral cannulation, providing another option in the approach to this complex pathologic condition.

We thank Kimberly Shockey for her statistical expertise used for this study and Sandra Burks for facilitating the institutional review board protocol and its approval.

\section{References}

1. Hirst AE, Johns VJ, Kime SW. Dissecting aneurysm of the aorta: a review of 505 cases. Medicine (Baltimore). 1958;37:217-79.

2. Hagan PG, Nienaber CA, Isselbacher EM, Bruckman D, Karavite DJ, Russman PL, et al. The International Registry of Acute Aortic Dissection (IRAD): new insights into an old disease. JAMA. 2000;283: 897-903.

3. Bavaria JE, Brinster DR, Gorman RC, Woo YJ, Gleason T, Pochettino A. Advances in the treatment of acute type A dissection: an integrated approach. Ann Thorac Surg. 2002;74:S1848,52; discussion S1857-63.

4. Gleason TG. Contemporary management of aortic dissection: introduction. Semin Thorac Cardiovasc Surg. 2005;17:213.

5. Gallo A, Davies RR, Coe MP, Elefteriades JA, Coady MA. Indications, timing, and prognosis of operative repair of aortic dissections. Semin Thorac Cardiovasc Surg. 2005;17:224-35.

6. Kazui T, Yamashita K, Washiyama N, Terada H, Bashar AH, Suzuki $\mathrm{T}$, et al. Impact of an aggressive surgical approach on surgical outcome in type A aortic dissection. Ann Thorac Surg. 2002;74:S1844,7; discussion S1857-63.

7. Kirklin JW, Kouchoukos NT. Kirklin/Barratt-Boyes cardiac surgery: morphology, diagnostic criteria, natural history, techniques, results, and indications. 3rd ed. Philadelphia: Churchill Livingstone; 2003. p. 1938, I-83.

8. Cohn LH, Edmunds LH. Cardiac surgery in the adult. 2nd ed. New York: McGraw-Hill; 2003. p. 1573.

9. Kaiser LR, Kron IL, Spray TL. Mastery of cardiothoracic surgery. Philadelphia: Lippincott-Raven Publishers; 1998. p. 976.

10. Cope JT, Tribble RW, Komorowski B, Tribble CG. A simple technique for retrograde cerebral perfusion during circulatory arrest. J Card Surg. 1996;11:65-7.

11. Strauch JT, Spielvogel D, Lauten A, Lansman SL, McMurtry K, Bodian CA, et al. Axillary artery cannulation: routine use in ascending aorta and aortic arch replacement. Ann Thorac Surg. 2004;78:103-8.

12. Fusco DS, Shaw RK, Tranquilli M, Kopf GS, Elefteriades JA. Femoral cannulation is safe for type A dissection repair. Ann Thorac Surg. 2004;78:1285-9.

13. Panos A, Murith N, Bednarkiewicz M, Khatchatourov G. Axillary cerebral perfusion for arch surgery in acute type A dissection under moderate hypothermia. Eur J Cardiothorac Surg. 2006;29:1036-9.

14. Olsson C, Thelin S. Antegrade cerebral perfusion with a simplified technique: unilateral versus bilateral perfusion. Ann Thorac Surg. 2006;81:868-74.

15. Veron S, Neri E, Buklas D, Pula G, Benvenuti A, Massetti M, et al. Cannulation of the extrathoracic left common carotid artery for thoracic aorta operations through left posterolateral thoracotomy. Ann Vasc Surg. 2004;18:677-84.

16. Reuthebuch O, Schurr U, Hellermann J, Pretre R, Kunzli A, Lachat M, et al. Advantages of subclavian artery perfusion for repair of acute type A dissection. Eur J Cardiothorac Surg. 2004;26:592-8.

17. Mazzola A, Gregorini R, Villani C, Di Eusanio M. Antegrade cerebral perfusion by axillary artery and left carotid artery inflow at moderate hypothermia. Eur J Cardiothorac Surg. 2002;21:930-1.

18. Mazzola A, Gregorini R, Villani C, Di Eusanio M. Antegrade cerebral perfusion by axillary artery and left carotid artery inflow at moderate hypothermia. Eur J Cardiothorac Surg. 2002;21:930-1.

19. Lijoi A, Scarano F, Dottori V, Parodi E, Casali G, Bartolozzi F. Stanford type A aortic dissection. A new surgical approach. Tex Heart Inst J. 1998;25:65-7.

20. Minatoya K, Karck M, Szpakowski E, Harringer W, Haverich A. Ascending aortic cannulation for Stanford type A acute aortic dissection: another option. J Thorac Cardiovasc Surg. 2003;125:952-3.

21. Westaby S, Katsumata T, Vaccari G. Arch and descending aortic aneurysms: influence of perfusion technique on neurological outcome. Eur J Cardiothorac Surg. 1999;15:180-5.

22. Robicsek F, Zimmern SH, Howe HR. Subintimal retrograde perfusion during repair of aortic dissection: a potential cause of disaster. Ann Vasc Surg. 1988;2:298-302.

23. Parr GV, Manley NJ, Williams DR, Montesano RM. Obstruction of the true lumen during retrograde perfusion of type I aortic dissections: a simplified solution. Ann Thorac Surg. 1980;30:495-8.

24. Schachner T, Nagiller J, Zimmer A, Laufer G, Bonatti J. Technical problems and complications of axillary artery cannulation. Eur J Cardiothorac Surg. 2005;27:634-7.

\section{Discussion}

Dr Robert C. Robbins (Stanford, Calif). I guess what your presentation really means is that you cannot expect any different outcome if you keep doing things the same way and so you are trying to make us think outside the box a little bit and consider central cannulation. I have just a couple of brief questions.

Did the date of the operations differ across the distribution of time, or was central cannulation evenly distributed across the study period?

Dr Reece. It was not evenly distributed. Central cannulation was used a little more in the more recent period, but Dr Tribble 
tells me that he has been using it since 1986. As some of the people like Dr Kern come on, they were using it a little more, and probably an increased number of the attendings used it as the study period went on.

Dr Robbins. That leads to the next comment. I suspect that Dr Tribble would probably be the one who uses it the most, Dr Kron maybe the least, and Dr Kern in the middle, so how does this algorithm really fit? Is there really a lot of preoperative planning, or would, say, Dr Tribble consider central cannulation as the "default" and use it unless he could not do so for some reason that you have alluded to?

Dr Reece. I think that for the most part all of them will use it. Dr Tribble is definitely more likely to use it as his default, but he is the one who came up with the algorithm and the idea that we could focus it on each particular patient and his or her characteristics.

Dr. Robbins. It is too bad we are not going to have other discussants because I am sympathetic to this technique, having used it a few times first because I could not get a cannula up because of severe atherosclerosis. I think it is actually a technique that can be used, but I think most everyone else would go to the axillary route. Particularly, as you alluded to, if you are going to have longer circulatory arrest cases, then the axillary route is preferable, but I would argue that the axillary is preferable anyway. I have rarely seen an axillary artery dissected or have so much atherosclerosis that it was not usable.
I have just one more comment about holding the cannula in. I have found that it is pretty messy and you have to figure out how you can actually get in the true lumen. Speaking with Dr Kern, it really probably does not matter if there is a large intimal tear.

The one real question is, you had no deaths and really very few complications in the central cannulated patients versus the peripheral, so why do you think that was, particularly the large number of perioperative myocardial infarctions that you saw in the peripheral patients? I don't know that you'll have an answer for this, but it was interesting and I think the main issue of this paper.

Dr Reece. I cannot really explain it from the preoperative data. I think it is probably more a function of the small number of patients in the study. As we continue with this algorithm, we may see the difference decline. I do not think there is anything intrinsic to either type of cannulation that would make myocardial infarction more likely to occur, but that we are seeing the limitations of a small study group.

Dr Robbins. I have just one last comment. Since Dr Tribble has been doing it for 20 years now, I do not really see why intimal mural hematoma would be a problem. If there is no communication and you know you are in the true lumen, then you can just perfuse down the true lumen and there is some advantage to going antegradely. Similarly with the clot in the false lumen, I think it is probably more problematic going retrogradely. Therefore, I would argue that most people would say that the axillary artery is the best way to go, and that is the way I would do it, but you could easily make an argument that you could go centrally for all cases. 